\title{
PROBLEMAS DE TRADUCCIÓN DIRECTA E INVERSA EN LA COMPOSICIÓN DE ADJETIVOS Y PARTICIPIOS EN LENGUA ALEMANA
}

\author{
Ana Medina Reguera
}

La composición en lengua alemana es uno de los grandes temas que ocupa a los traductores de alemán prácticamente a diario. Existen dos problemas fundamentales: a la hora de traducir compuestos a la lengua española, debemos buscar una alternativa de tipo analítico o perifrástico para expresar el contenido del compuesto; en cuanto a la traducción español-alemán, es necesario conocer si el compuesto puede ser elaborado ad hoc, y en ese caso cuál es su escritura correcta, pues es bien sabido que la capacidad de composición en la unión de ciertas categorías léxicas ha visto recortada sus posibilidades a partir de la reforma ortográfica alemana de $1996^{1}$. En el presente trabajo se abordan estas dos cuestiones, que se aplican a las uniones de base adjetiva, cuyo primer miembro también es un adjetivo. La palabra base podrá ser un adjetivo de lengua o un participio de presente o de perfecto, desempeñando la función sintáctica de adjetivo.

Los mecanismos de formación de nuevas palabras en lengua alemana son muy variados, del mismo modo que su terminología y sus innumerables clasificaciones en gramáticas y manuales de morfología alemana ${ }^{2}$. Existe, no obstante, consenso general a la hora de distinguir cuatro tipos fundamentales: la composición, en la que se unen formas complejas con raíces propias (Fensterrahmen, Sprachwissenschaft); la prefijación, en la que un elemento de menor rango se antepone a la raíz (Missmut, Unmut, verpassen); la sufijación, en la que un elemento es pospuesto a la raíz (mutig, Sicherheit, rötlich); y la conversión, que puede definirse como un mecanismo que pročura el cambio de categoría léxica sin marcas morfológicas, como ocurre por ejemplo en la nominalización de verbos (das Lesen) o de adjetivos (der Alte, die Verliebte, der Beste).

Evidentemente, esta simplificación es tan grande, que hay que añadir primeramente, que multitudes de palabras alemanas presentan varios mecanismos al mismo tiempo: Versicherung, verständnissvoll, das Unvergessene, bergsteigen, etc., son ejemplos de la interacción de mecanismos de formación de palabras y de una morfología rica en flexión y de carácter sintético.

\footnotetext{
${ }^{1}$ Sobre la tendencia a la separación gráfica véase Nerius, Dieter (2000): Deutsche Orthographie. Mannheim: Dudenverlag, págs. 146-173 y Utz Maas (2000): Orthographie. Materialien zu einem erklärenden Handbuch zur Rechtschreibung des Deutschen. Osnabrück, págs. 223-247.

2 Véase al respecto entre otros Duden (1998): Grammatik der deutschen Gegenwartsprache. Mannheim. 6 ed (págs. 419-440); Fleischer, Wolfgang (1971): Wortbildung der deutschen Gegenwartssprache. Leipzig: Bibliographisches Institut; Fleischer, Wolfgang/ Barz, Irmhild (1992): Wortbildung der deutschen Gegenwartssprache. Tübingen.
} 
En este contexto tan amplio, se quiere limitar la temática a la traducción de adjetivos complejos. Esto significa, adjetivos que se unen a otros adjetivos. En mi opinión, puede ser útil partir de la diferenciación de varios tipos, que he clasificado del siguiente modo:

1.

Unión de dos adjetivos que pueden aparecer según el contexto o bien unidos, o bien cada uno por separado. Un adjetivo calificativo como por ejemplo dunkel (Ich möchte meine Haare dunkel färben) se une a otro adjetivo (Du solltest deine Haare rot färben), para formar un adjetivo compuesto (Ich habe dunkelrote Haare). Otros ejemplos son: bitterböse, blaugrau, dummdreist, nasskalt, taubstumm

2. Unión de dos adjetivos en los que uno de los dos miembros depende del otro de tal modo, que no puede aparecer aisladamente: letzmalig, blauäugig, rothaarig, hellhäutig, schwerstbehindert, großspurig, halbstündich, halbstündlich ("malig, *aügig, *häutig, *schwerst, etc.)

3. Unión de dos adjetivos, siendo el segundo de ellos un participio de presente o de perfecto. El participio desempeña en estos casos la función gramatical de un adjetivo. El primer miembro modifica al segundo: schwerbewaffnet, schwerwiegend, vollbeschäftigt, volltönend

Como norma general, puede decirse que el primer miembro del compuesto realiza la función de ampliar, reforzar, delimitar o debilitar de algún modo el significado del segundo. Para la traducción es fundamental el hecho de que el primer miembro es siempre el modificador (por ejemplo en schwerbewaffnet schwer modifica a bewaffnet), mientras que el segundo elemento de la composición es el miembro modificado. Además, debemos tener en cuenta los siguientes dos factores importantes:

El primer miembro de los compuestos es de base adjetiva, aunque tiene características especiales que permiten formar largas filas de formaciones distintas. No será aquí objeto de estudio su condición gramatical, y aunque algunos de estos adjetivos han sido analizados en numerosas ocasiones como prefijoides (en al. Präfixoide o Halbpräfixe) ${ }^{3}$, no todos los adjetivos pueden analizarse de este modo, debido a su elevado contenido semántico. La denominación de prefijoide se relaciona con procesos de lexicalización semántica y de gramaticalización:

"Durch Grammatikalisierung entstehen aus selbständigen Stämmen sog. Halbaffixe oder Affixoide wie frei in schadstoffrei, angstfrei, kostenfrei, wie mäßig in handlungsmäßig, fabrikmäßig, turnusmäßig oder wie hoch in hochmodern, hochmotiviert. Sie haben noch die Form des selbständigen Stammes, aber nicht mehr die zugehörige "normale" Wortbedeutung. Ihre Bedeutung ist abstrakter. Sie neigen zu Reihenbildung und können sich durchhaus zu echten Derivationsaffixen entwickeln"A

En segundo lugar, cuando el segundo miembro del compuesto es un participio, sea de presente o de perfecto, éste desempeña la función de adjetivo. La realización como adjetivo le permite acoger la morfología de flexión adjetiva, lo cual no le exime de su flexión original como participio (schwerbeschädigt-e Frau, enganliegend-er Rock).

\footnotetext{
${ }^{3}$ Es así por ejemplo en Lachachi, D.E. (1992): Halbaffigierung: Eine Entwicklungstendenz in der deutschen Wortbildung. En: Info DAF 19 (págs. 36-43) o en Motsch, W (1996): Affixoide. Sammelbezeichnung für Wortbildungsphänomene oder linguistische Kategorie? En: Deutsch als Fremdsprache 33 (págs. 160-168) o en Vögeding, J (1981): Das Halbsuffix ‘-frei’. Zur Theorie der Wortbildung. Tübingen.

${ }^{4}$ Eisenberg, Peter: Grundriss der Deutschen Grammatik. Das Wort. Stuttgart: Metzler 1998, pág. 206
} 
A continuación hemos tomado los compuestos formados con los primeros miembros schwer, eng y voll:

$\begin{array}{ll}\begin{array}{l}\text { Compuestos formados por el adjetivo -schwer y otros adjetivos o participios: } \\ \text { schwerbelastet }\end{array} & \text { muy cargado (fig. abrumado) } \\ \text { schwerbehindert } & \text { muy impedido, gravemente discapacitado } \\ \text { schwerbeladen } & \text { muy cargado } \\ \text { schwerbeleidend } & \text { muy ofendida/o } \\ \text { schwerbepackt } & \text { muy cargado } \\ \text { schwerbeschädigt } & \text { gravemente mutilado } \\ \text { schwerbetrunken } & \text { muy borracho, borracho como una cuba } \\ \text { schwerbewaffnet } & \text { armado hasta los dientes, fuertemente armado } \\ \text { schwerblütig } & \text { serio, grave, lento, tardo } \\ \text { schwerentäuscht } & \text { cruelmente/seriamente desilusionado } \\ \text { schwererziehbar } & \text { difícil de educar } \\ \text { schwerfällig } & \text { torpe, lento, tardo de entendimiento } \\ \text { schwerflüssig } & \text { viscoso } \\ \text { schwergewichtig } & \text { de mucho peso, obeso } \\ \text { schwerhörig } & \text { un poco sordo, duro de oído } \\ \text { schwerkrank } & \text { gravemente enfermo } \\ \text { schwerlöslich } & \text { difícilmente soluble } \\ \text { schwermutig } & \text { melancólico, hipocondríaco } \\ \text { schwerreich } & \text { muy rico } \\ \text { schwerverdaulich } & \text { indigesto } \\ \text { schwerverkäuflich } & \text { de difícil venta } \\ \text { schwerverletz } & \text { gravemente herido } \\ \text { schwerverständlich } & \text { difícil de comprender } \\ \text { schwerverträglich } & \text { dificilmente digestible, digerible, persona dificil de tratar, muy } \\ \text { poco sociable } & \text { con heridas graves } \\ \text { schwerverwundet } & \text { muy serio, decisivo, de gran importancia, de gran trascendencia } \\ \text { schwerwiegend } & \end{array}$

Como puede observarse, las traducciones del adjetivo schwer, que al ser modificadores del segundo miembro suele traducirse como adverbio, pueden ser muy variadas: pesadamente, difícilmente, gravemente, seriamente, severamente, peligrosamente, rigurosamente, mucho, muy, etc.

Como suele ocurrir con las palabras compuestas de dos componentes, el primer componente es el que lleva el acento principal de la palabra. El acento marca la diferencia entre los compuestos y otros sintagmas homólogos: 
(1) Ein schwerreicher Mann

(1a) Der Mann ist schwer reich

Este cambio de acentuación se produce en todos los casos en los que el compuesto tiene un sintagma idéntico a él, aunque en la mayoría de los casos esto no parece producir diferencias en la traducción:

(2) ein schwererziehbares Kind

$=$ un niño difícil de educar

(3) Das Kind war schwer erziehbar

= El niño era difícil de educar / Era difícil/complicado educar al niño

Algunos autores, sin embargo, opinan que la diferente acentuación podría estar ligada a matices diferentes de significado. Eisenberg realiza el siguiente análisis: ${ }^{5}$

(4) Er ist schwer behíndert

(4a) Er ist schwérbehindert

En el primer caso se trata de un sintagma en el que el adjetivo que está en primer lugar es atributo del segundo. El segundo elemento, núcleo del sintagma, lleva el acento. En el segundo caso, el acento lo lleva el primer miembro con motivo de su diferente significado. En er ist schwer behíndert se trata en primer lugar de la comunicación-información de que existe una discapacidad o minusvalía. En segundo lugar se sitúa la información por la que se nos dirá de esa minusvalía que es grave. En la segunda frase, er ist schwérbehindert, la palabra correspondiente se encarga de antemano de comunicarnos el concepto de una grave minusvalía (eine Schwerbehinderung). Su aplicación se daría en un un caso hipotético en el que en la conversación ya se ha hablado de una minusvalía y ahora se trata de su diferenciación o concrección. Este ejemplo en particular de schwerbehindert es una palabra que ya está lexicalizada y terminologizada. Se aplica -del mismo modo que en el caso de la palabra schwerbeschädigt- para aquellas personas cuyo impedimento se corresponde con unos criterios ya establecidos en el ámbito jurídico y civil.

Compuestos formados por el adjetivo eng- y otros adjetivos o participios:

$\begin{array}{ll}\text { enganliegend } & \text { ajustado, ceñido } \\ \text { engbedruckt } & \text { compacto, de impresión compacta } \\ \text { engbefreundet } & \text { ser amigos íntimos } \\ \text { engbegrenzt } & \text { muy limitado } \\ \text { engbeschrieben } & \text { con letra muy apretada } \\ \text { engbrüstig } & \text { estrecho de pecho } \\ \text { engherzig } & \text { estrecho de miras, mezquino, poco generoso } \\ \text { engliiert } & \text { estar estrechamente relacionados } \\ \text { engmaschig } & \text { de malla tupida } \\ \text { engspaltig } & \text { apretado, estrechamente espaciado }\end{array}$

\footnotetext{
${ }^{5}$ Eisenberg 1998, op. cit. pág. 318
} 


$\begin{array}{ll}\text { engstirnig } & \text { de cortas luces, obcecado, estrecho de miras } \\ \text { engumgrenzt } & \text { que limita estrechamente con algo, estrechamente delimitado } \\ \text { engverbunden } & \text { estrechamente unidos } \\ \text { engverwandt } & \text { ser pariente cercano }\end{array}$

La traducción del primer componente eng tampoco es constante, y puede variar de estrechamente hasta muy: Er hat einen engbegrenzten Horizont = tiene una visión muy limitada. En otros casos no es necesario traducir el primer miembro: Sie trägt immer enganliegende Kleider = Ella siempre lleva vestidos ajustados/ceñidos .

En algunos çasos sólo se encuentra el sentido figurado, no siendo posible la traducción literal: engstirnig \pm estrecho de miras pero no *estrecho de frente; engherzig = mezquino, pero no *estrecho de corazón. Se ha encontrado sin embargo un compuesto que solo admite el significado literal: engbrüstig.

\begin{tabular}{ll}
$\begin{array}{ll}\text { Compuestos formados por el adjetivo voll y otros adjetivos o participios: } \\
\text { vollautomatisch }\end{array}$ & completamente automático \\
vollautomatisiert & completamente automatizado \\
vollbärtig & barbudo, con barba \\
vollbeladen & a plena carga \\
vollberechtigt & con pleno poder \\
vollbeschäftigt & trabajar la jornada completa, estar muy atareado \\
vollbesetz & totalmente repleto/ ocupado \\
vollblütig & pletórico \\
vollbusig & de pechos grandes \\
vollendet & acabado, cumplido, comsumado \\
vollentwickelt & plenamente desarrollado \\
vollgefressen & atracado, hinchado de comer \\
vollgefüllt & completamente lleno \\
vollgegessen & harto de comer \\
vollgeladen & completamente cargado \\
vollgepackt & completamente cargado \\
vollgepfroft & totalmente repleto \\
vollgepumpt & inflado \\
volltrunken & completamente borracho \\
vollgeschlagen & atracado, hinchado de comer \\
vollgeschmiert & embadurnado, lleno de garabatos \\
vollgeschrieben & completamente rellena (una hoja, un papel) \\
voltgespritzt & salpicado \\
vollgestopft & atiborrado, completamente lleno \\
\hline
\end{tabular}




$\begin{array}{ll}\begin{array}{l}\text { vollgültig } \\ \text { volljährig } \\ \text { vollklimatisiert }\end{array} & \begin{array}{l}\text { perfectamente válido, irrecusable } \\ \text { mayor de edad } \\ \text { vollkommen } \\ \text { vollleibig }\end{array} \\ \text { totalmente climatizado } \\ \text { pollmundig } & \text { gordo, obeso } \\ \text { vollschlank } & \text { con mucho sabor, fanfarrón } \\ \text { vollständig } & \text { metido en carnes, rellenito/a } \\ \text { vollstreckbar } & \text { completo, íntegro } \\ \text { volltönend } & \text { ejecutable, ejecutorio } \\ \text { vollwertig } & \text { sonoro, armonioso }\end{array}$

En cuanto a las traducciones posibles con voll como primer adjetivo, debemos destacar algún término lexicalizado en un ámbito semántico concreto, como ocurre con el término jurídico vollstreckbar, así como algún término que se contradice en su significado literal (vollschlank). Es significativo que hay términos en los que no se necesita traducir el adjetivo voll porque en alemán su significado está implícito y no se utilizan estos vocablos sin el primer miembro del compuesto, es deir, vollgepumpt, volltönend, vollleibig, volljährig, etc., pero no *Die Reifen sind gepumpt, *Das Kind ist leibig, *Der Mann ist jährig, etc. Algunos compuestos tienen doble significado. La composición vollmundig, en su significado literal significa "con mucho sabor" (también en el contexto vinícola, Der Wein ist vollmundig), mientras que en significado figurado significa "fanfarrón". Por último, abundan las expresiones relacionadas con la comida: vollgegessen, vollgefressen, vollgeschlagen(-er Bauch) con el significado de estar lleno, que se ha dado el atracón, que se ha hinchado de comer, etc.

En general, observamos una serie de características generales que se repiten en las largas filas que hemos presentado. Siguiendo criterios semánticos, en los adjetivos de los ejemplos recogidos el significado de la segunda parte se ve modificado, en el sentido de reforzamiento o debilitamiento, por una raiz adjetiva.

Aunque no han sido recogidos, la raiz modificadora puede en general ser también un superlativo (schwerstbehindert, engstverbunden).

En posición predicativa, el adjetivo puede desempeñar la función sintáctica de adjetivo o de adverbio:

Diese Maschine ist automatisch.

Diese Maschine läuft automatisch.

En español hay que distinguir en cambio entre dos diferentes traducciones:

Esta máquina es automática (adjetivo).

Esta máquina funciona automáticamente/de manera automática (adverbio).

Así lo indican también Gil/Banus cuando afirman:

"Die [...] erörtete formale Gleichheit für das prädikative Adjektiv und das modale Adverb ist ein Zeichen dafür, daß die Funktion des Adjektivs und des Adverbs letztlich die 
gleiche ist: zu bestimmen, zu bezeichnen, zu kennzeichnen. Die ersten beziehen sich auf ein Substantiv, die zweiten auf ein Verb, ein Adjektiv oder ein anderes Verb"

Esto influye a la hora de introducir un modificador:

Die Maschine ist vollautomatisch.

=Esta máquina es totalmente automática

Diese Maschine läuft vollautomatisch

$=$ *Esta máquina funciona totalmente automáticamente

$=$ ?Esta máquina funciona de manera totalmente automática/de modo totalmente automático

Hasta este punto hemos presentado un tratamiento de la cuestión partiendo de los compuestos en lengua alemana. A partir de aquí van a ser tratadas estas composiciones desde el punto de vista contrario, la traducción del español al alemán.

En cuanto a los problemas de traducción español-alemán, se pueden presentar en mi opinión las siguientes dificultades:

1. Dificultad en torno a la posibilidad o imposibilidad de crear un compuesto del tipo que estamos tratando (ad hoc).

2. Dificultad en torno a una posible escritura como compuesto, con la consecuente unión gráfica de palabras (en alemán Zusammenschreibung) o como sintagma, con la consecuente separación ortográfica (en alemán Getrenntschreibung).

Aunque los dos puntos están estrechamente relacionados, el primero de ellos concierne más al carácter sintético de la lengua alemana y sus delicados límites en el lenguaje escrito. Dada la altísima productividad de ciertos adjetivos y siguiendo el modelo de los ejemplos citados anteriormente, podemos construir infinidades de nuevas palabras y escribirlas en principio como una palabra compuesta, por ejemplo en oraciones de la lengua coloquial como Der Mann ist schwerböse, aber immer, noch vollruhig o Der Mann ist schwerbetrunken, aber trozdem noch vollaktiv, y así sucesivamente.

En cuanto a la segunda dificultad, la ortografía se encarga en cada caso de regular los procesos de unión gráfica (como consecuencia de una composición) o separación gráfica (en el caso de dos unidades sintácticas independientes). La cuestión es delicada y las opiniones de los miembros de las comisiones ortográficas de los países de lengua alemana son diversas. Tras los intensos debates y discusiones en torno a estos casos con motivo de la reforma ortográfica de 1996, se ha reformado en gran medida la ortografía de estos compuestos, basándose sobre todo en criterios de lexicalización semántica.

Después de la reforma se tomó tras numerosas discusiones la decisión de dejar como uniones ortográficas las siguientes:

schwerbeschädigt, schwerfällig, schwerbehindert y schwerwiegend vollbärtig, vollbeschäftigt, vollblütig, vollelastisch, vollelektronisch, vollendet, vollgefressen, volljährig, vollklimatisiert, vollkommen, vollmundig, vollreif, vollschlank, vollspurig, vollständig, volltönend, volltönig, vollwertig, vollwichtig, vollzählig, vollziehbar engstirnig, engherzig, engbrüstig

Esto significa que todos los demás se escriben tras la reforma como sintagma (GZ). 
La razón que nos ofrece el reglamento ortográfico para explicar estos cambios es la siguiente: Deben ser escritos como sintagma aquellas formaciones que tienen la capacidad de ser ampliadas en grado de comparación e intensidad ${ }^{6}$ :

Antes:

- Das ist eine schwerverständliche Sprache

- Der Mann war schwerbewaffnet

Ahora:

- Das ist eine schwer verständliche Sprache (ya que es posible: schwerer verständlich).

- Der Mann war schwer bewaffnet (der andere war noch schwerer bewaffnet).

El cambio de grado de intensidad puede producirse también por medio de adverbios:

Antes:

- Das ist mir schwergefallen.

- Das sind dünnbevölkerte Landstriche.

- Es war ernstgemeint.

Ahora:

- Das ist mir sehr gefallen (sehr schwer gefallen).

- Das sind dünn bevölkerte Landstriche (besonders dünn bevölkert).

- Es war ernst gemeint (sehr ernst gemeint).

Desafortunadamente, este criterio no se puede aplicar a todas las composiciones por igual. He tomado del diccionario ortográfico anterior a la reforma (Rechtschreibduden 1991) algunos de los compuestos con halb:

$\begin{array}{ll}\text { halbblind } & \text { medio ciego } \\ \text { halbbürtige } & \text { hermanos de un sólo vínculo } \\ \text { halbdurchlässig } & \text { semipermeable } \\ \text { halbfertig } & \text { a medio hacer } \\ \text { halbgar } & \text { medio cocido } \\ \text { halbgeschlossen } & \text { entreabierto } \\ \text { halbjährlich } & \text { semestral } \\ \text { halbleer } & \text { medio vacio } \\ \text { halbmilitärisch } & \text { paramilitar } \\ \text { halbnackt } & \text { medio desnudo } \\ \text { halboffen } & \text { medio abierto } \\ \text { halbseitig } & \text { de un solo lado, halbseitige Lähmung, hemiplejia } \\ \text { halbstündig } & \text { de media hora }\end{array}$

\footnotetext{
${ }^{6}$ Vid. § 36 E 1 (4) del Duden 1: Die deutsche Rechtschreibung 1996 (pág. 876)
} 
Problemas de traducción directa e inversa en la composición...

$\begin{array}{ll}\text { halbstündlich } & \text { cada media hora } \\ \text { halbverhungert } & \text { medio muerto de hambre } \\ \text { halbvoll } & \text { medio lleno } \\ \text { halbwach } & \text { medio despierto }\end{array}$

El criterio semántico que ha seguido la reforma respecto a estas construcciones se basa en los posibles significados de halb:

1. Uno de los significados es "en oposición a ganz". En ese caso se escribirá como sintagma:

- Er hat mich wohl nur halb verstanden.

- Er war erst halb angezogen.

- $\quad$ Eine halb leere (antes halbleere) Flasche

- $\quad$ Ein halb offene (antes halboffene) Fenster

- $\quad$ Ein halb verhungerter (antes halbverhungerter) Vogel

2. También se utiliza la escritura como sintagma cuando halb significa "en parte" ("teils"):

- Sie machte ein halb freundliches, halb ernstes Gesicht.

3. Un tercer significado lo define el reglamento ortográfico del siguiente modo: "Zusammenschreibung, wenn halb als bedeutungsabschwächender Zusatz aufgefasst wird“"

- $\quad$ ein halbhoher (nicht sehr hoher) Zaun

- $\quad$ halbbittere (nicht sehr bittere) Schokolade

4. Para terminar con este pequeño caos, ningún autor expliça por qué se permiten las dos posibilidades en los siguientes casos:

- halbgares Fleisch / halb gares Fleisch

- halblinks stehen / halb links stehen

- halbfertig/halb fertig

- halbblind/halb blind

- halbreif/halb reif

- halbfest/halb fest

O sea, que halb gar puede significar "nicht sehr gar" (Zusammenschreibung) o "nicht ganz gar" (Getrenntschreibung) pero otros ya lexicalizados, como halbbitter o halbblind, no pueden significar "nicht ganz bitter" ni "nicht ganz blind", sino que simplemente significan "nicht sehr blind/bitter".

En este punto tengo que decir, desgraciadamente, que la regulación de estos compuestos es a mi parecer, bastante mejorable. Las regularidades que ofrece el criterio semántico no son consecuentes ni sistemáticas en este ámbito, así que regular su ortografía siguiéndolo ha sido, en mi opinión, algo precipitado y quizás erróneo. De la misma opinión son Utz

${ }^{7}$ Rechtschreibduden (1996:333), haciendo referencia a $§ 36$ (5), en la pág. 876 del mismo. 
Maas, Hartmut Günther o Peter Eisenberg. El principal problema de marcar el significado a través de la unión o la separación gráfica, es que a la vista de un compuesto aislado, no siempre es fácil encuadrarlo en uno de los significados que el reglamento ortográfico propone. Por si no hubiera sido bastante transparente en los compuestos con el primer miembro halb, a continuación se demostrará esto una vez más con los compuestos cuyo primer adjetivo es hoch: ${ }^{8}$

\section{"II. Schreibung in Verbindung mit Adjektiven od. Partizipien}

1. Getrenntschreibung, wenn »hoch « relativ gebraucht wird, d.Ch. erweiterbar od. steigerbar ist:

eine [sehr] hoch stehende/hoch gestellte [alte Schreibungen hochstehende, hochgestellte] Persönlichkeit; aber eine hochgestellte Zahl

[sehr] hoch gesteckte [alte Schreibung hochgesteckte] Ziele; aber hochgesteckte Haare

hoch besteuerte Einkommen; ein hoch bezahlter Job; hoch dotierte Architektinnen; hoch empfindliches Filmmaterial; eine hoch gewachsene Pflanze; hoch qualifizierte Akademiker usw. [alte Schreibungen hochbesteuert, hochbezahlt, hochdotiert, hochempfindlich, hochgewachsen, hochqualifiziert, usw.]

\section{Zusammenschreibung}

a) wenn »hoch « absolut gebraucht wird, d. Ch. nicht enweiterbar od. steigerbar ist:

ein hochgeschlossenes Kleid

hochgekurbelte (geschlossene) Fenster

hochtrabende/hochfliegende Pläne

hochgestochen reden

b) wenn »hoch « als Richtungsangabe gebraucht wird:

hochgesteckte Haare; hochfliegende Späne usw.

c) wenn »hoch « rein intensivierend gebraucht wird:

hochanständig (sehr anständig), hochberühmt, hocherfreut, hochfahrend, hochglänzend, hochtrabend, hochbeinig, hochbekommen, hochberühmt, hochbetagt, usw.

In Zweifelsfällen ist sowohl Getrennt- als auch Zusammenschreibung möglich:

eine hochbegabte, auch hoch begabte/hochgebildete, auch hoch gebildete Frau"

El lecțor apreciará sin duda la dificultad de estas reglas y lo confuso de las mismas. Si tenemos que decidir, después de haber "asimilado" la información anterior, cuál es la ortografía para cualquiera de estas formaciones que se presentan a continuación (y que no aparecen como ejemplos en las explicaciones anteriores de la vigésimo segunda edición del Duden, el lector podrá ver rápidamente que no es siempre fácil adjudicarles el significado presentado arriba como $a, b, \mathrm{o} c$.:

hochnäsig, hochmutig, hochmodern, hochmodisch, hochpreisig, hochprozentig, hochräderig(er) Wagen, hochschwanger, hochsinnig, hochstämmig,

\footnotetext{
${ }^{8}$ Tomado de PC-Bibliothek Express 2.1, Revision 13 (Software), Bibliographisches Institut \& F.A. Brockhaus AG, Mannheim 2000
} 
hochverdient, hochverehrt (in der Anrede auch hochverehrtest), hochverzinslich, hochwilkommen, hochwirksam

\section{CONCLUSIONES}

Tras la reforma ortográfica se limita la capacidad de formación de palabras, puesto que la tendencia que ésta ha seguido en su proceso de regulación es la separación gráfica y no la unión de palabras. Esto ocurre también con estos compuestos adjetivos que hemos analizado en este trabajo. Sintácticamente esto se traduce en procesos de degramaticalización, y semánticamente, se limita la lexicalización de compuestos a cambios de significado tras la unión de ambos (es decir, siguiendo la tendencia que se ha seguido con los verbos: hoch gehen=subir, pero hochgehen=explotar).

Para conocer el modo de escritura de estos compuestos, es importante a mi parecer conocer en primer lugar tres reglas sencillas:

1. Las composiciones de adjetivos del mismo rango se escribirán siempre unidas: blaugrau, dummdreist, feuchtwarm, grünblau, nasskalt, taubstumm, etc.

2. Las composiciones en las que la ampliación por medio de adverbios o cambio de grado es posible se escribirán por separado: schwer bewaffnet, schwer verständlich, voll gegessen, hell leuchtend, schwach bevölkert, eng verwandt, etc.

3. Las composiciones en las que la ampliación por medio de adverbios o cambio de grado no es posible se escribirán unidas: hellwach, hellhörig, schwachsichtig, schwarzköpfig, schwerwiegend, engstirnig

No obstante, a posteriori estas tres regularidades no son suficientes, dada la dificultad que el criterio semántico le aporta a la ortografía, así como los procesos de lexicalización y gramaticalización que afectan a muchas de estas construcciones y cuyo proceso no ha terminado aún.

Los compuestos con un primer componente halb o hoch tienen, en mi opinión, una ortografía complicada porque siguen criterios únicamente semánticos y no es fácil para el usuario de la lengua decidir sobre ellos a la hora de escribirlos.

\section{BIBLIOGRAFÍA:}

BERTELSMANN LEXIKON (1996): Bearbeitet von Hermann Ursula und Götze Lutz. München: Lexikographisches Institut

DUDEN (1986): Rechtschreibung der deutschen Sprache. Mannheim.19 ed.

DUDEN (1991): Rechtschreibung der deutschen Sprache. Mannheim.20 ed.

DUDEN (1996): Rechtschreibung der deutschen Sprache. Mannheim.21 ed.

DUDEN (1998): Grammatik der deutschen Gegenwartsprache. Mannheim. 6 ed.

DUDEN (1998): Grammatik der deutschen Gegenwartsprache. Mannheim. 6 ed (págs. 419-440)

EISENBERG (1998): Grundriss der deutschen Grammatik. Das Wort. Stuttgart, Weimar: Metzler

FLEISCHER, WOLFGANG (1971): Wortbildung der deutschen Gegenwartssprache. Leipzig: Bibliographisches Institut 
FLEISCHER, WOLFGANG/ BARZ, IRMHILD (1992): Wortbildung der deutschen Gegenwartssprache. Tübingen

Gil, Alberto/Banus, Enrique (1991): Kommentierte Übersetzung Deutsch-Spanisch. $4^{\text {a }}$ Ed. Romanistischer Verlag, Bonn

INTERNATIONALER ARBEITSKREIS FÜR ORTHOGRAPHIE (1995): Deutsche Rechtschreibung.

LACHACHI, D.E. (1992): Halbaffigierung: Eine Entwicklungstendenz in der deutschen Wortbildung. En: Info DAF 19, págs. 36-43

LANGENSCHEIDTS HANDWÖRTERBUCH SPANISCH-DEUTSCH (I), Deutsch-Spanisch (II). Langenscheidts: Berlin 1984

MOTSCH, W (1996): Affixoide. Sammelbezeichnung für Wortbildungsphänomene oder linguistische Kategorie? En: Deutsch als Fremdsprache 33, págs. 160-168

NERIUS, DIETER (2000): Deutsche Orthographie. Mannheim: Dudenverlag, págs. 146173

PC-BIBLIOTHEK EXPRESS 2.1, Revision 13 (Software), Bibliographisches Institut \& F.A. Brockhaus AG, Mannheim 2000

REGELN UND WÖRTERVERZEICHINIS. VORLAGE FÜR DIE AMTLICHE REGELUNG. Tübingen: Günther Narr

UTZ MAAS (2000): Orthographie. Materialien zu einem erklärenden Handbuch zur Rechtschreibung des Deutschen. Osnabrück, págs. 223-247

VÖGEDING, J. (1981): Das Halbsuffix '-frei'. Zur Theorie der Wortbildung. Tübingen. 